\title{
Simulación computacional del desempeño de un sistema fotovoltaico acoplando generadores termoeléctricos y reflectores difusos de refuerzo
}

\author{
Computer simulation of the performance of a photovoltaic system by coupling thermoelectric generators and boost \\ reflectors
}

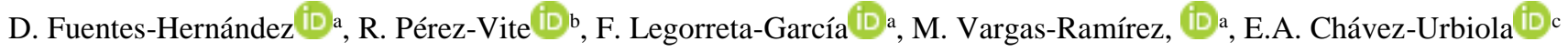 \\ a Área Académica de Ciencias de la Tierra y Materiales, Universidad Autónoma del Estado de Hidalgo, 42184, Pachuca, Hidalgo, México. \\ ${ }^{b}$ Área Académica de Matemáticas y Física, Universidad Autónoma del Estado de Hidalgo, 42184, Pachuca, Hidalgo, México. \\ ${ }^{c}$ CONACyT-UAEH, Universidad Autónoma del Estado de Hidalgo, 42184, Pachuca, Hidalgo, México.
}

\begin{abstract}
Resumen
En el presente trabajo se muestra un estudio preliminar de la generación de energía en paneles de silicio monocristalino en cinco condiciones de operación: ASTMG-173-03, $1000 \mathrm{~W} / \mathrm{m}^{2} \sin$ enfriamiento, $1000 \mathrm{~W} / \mathrm{m}^{2}+$ generadores termoeléctricos (TEG), acoplamiento de un panel + reflector difuso de refuerzo y acoplamiento de un panel + reflector difuso de refuerzo + TEG. El estudio se realizó comparando los resultados obtenidos mediante la simulación computacional de estos sistemas en MatLab Simulink, donde se analizaron cinco variables de interés, irradiancia, temperatura de operación, diferencia de potencial, intensidad de corriente eléctrica y potencia generada, determinando la viabilidad de la integración de reflectores difusos de refuerzo fabricados a partir de una película de base cerámica a sistemas solares fotovoltaicos.
\end{abstract}

\section{Palabras Clave:}

Generadores termoeléctricos, reflector difuso de refuerzo, simulación de sistemas solares fotovoltaicos, radiación solar, temperatura de operación.

\begin{abstract}
In the present work a preliminary study of energy generation in monocrystalline silicon panels is shown in five operating conditions: ASTMG-173-03, $1000 \mathrm{~W} / \mathrm{m}^{2}$ without cooling, $1000 \mathrm{~W} / \mathrm{m}^{2}+$ thermoelectric generators (TEG), coupling of a panel + reinforcing boost reflector and coupling of a panel + reinforcing boost reflector + TEG. The study was carried out comparing the results obtained through the computational simulation of these systems in MatLab Simulink, where five variables of interest were analyzed, irradiance, operating temperature, potential difference, intensity of electric current and generated power, determining the viability of the integration of boost reflector made from a ceramic-based film to photovoltaic solar systems.
\end{abstract}

Keywords:

Thermoelectric generators, boost reflector, solar photovoltaic systems simulation, solar radiation, operation temperature.

\section{Introducción}

El avance científico y tecnológico en el último siglo ha permitido que ramas como la medicina, agricultura, energía, entre otros, tengan un desarrollo acelerado, lo que permitió mejorar la calidad de vida, esto provocó un enorme crecimiento en el número de habitantes en el planeta. En la Figura 1 podemos observar como a partir del año 1900, el crecimiento de habitantes es increíblemente rápido en comparación con el de años anteriores, por lo tanto también su demanda de energía, principalmente en forma de energía eléctrica, sin embargo, las tecnologías existentes no fueron lo suficientemente eficientes para satisfacer la demanda y poder generar un crecimiento energético sustentable debido a estar basadas en el aprovechamiento del petróleo y sus derivados. Esto dio lugar a que en la década de 1970 el mundo cayera en una crisis energética, que provoco que la demanda de los combustibles fósiles fuese superior a la oferta, de acuerdo a la regla de oferta y demanda, el costo del crudo aumentó en los próximos años, este aumento fue tan drástico que de su valor nominal que rondaba los 20 dólares alcanzo a doblar su precio como se muestra en la Figura 2. (ECLAC, 1988.)

\footnotetext{
*Autor para la correspondencia: chavez.urbiola@gmail.com (Edgar A. Chávez-Urbiola) Correo electrónico: demetrio.fuentes.hdez@gmail.com (Demetrio Fuentes-Hernández), soul.vite.rafael@hotmail.com (Rafael Pérez-Vite), felegorreta@hotmail.com (Felipe
} Legorreta-García), marissav@uaeh.edu.mx (Marissa Vargas-Ramírez) 
Esto último, motivo la búsqueda de nuevas fuentes de energía sustentables que permitan alcanzar un desarrollo sostenible, entre estas formas de aprovechamiento energético que buscan cubrir la demanda cada vez más grande de energía de la población mundial, destaca la energía solar, las aplicaciones de este recurso son principalmente el calentamiento de agua y la generación de energía eléctrica mediante paneles fotovoltaicos.

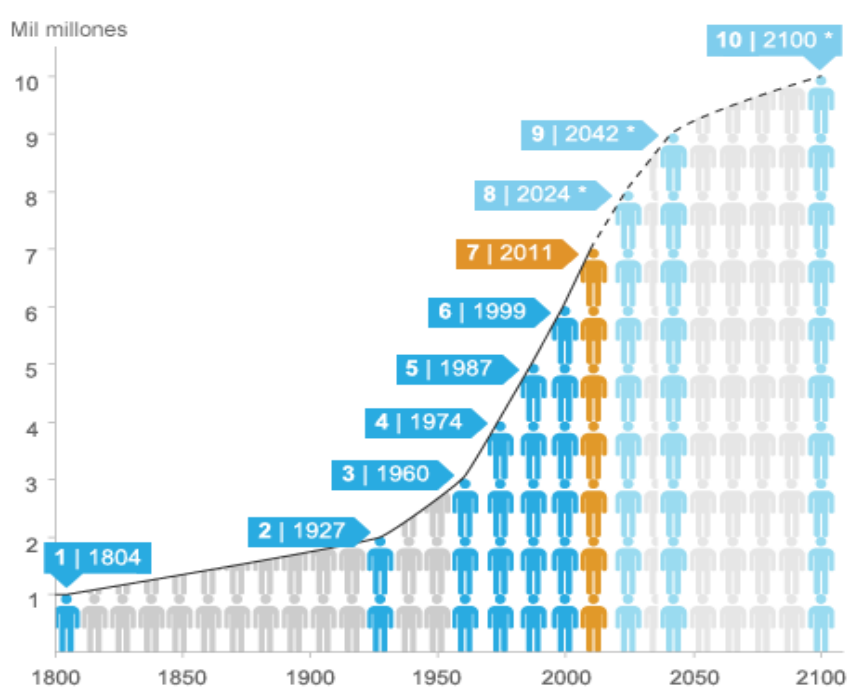

Figura 1: Crecimiento poblacional. Fuente: Fondo de población de la $\mathrm{ONU}$.

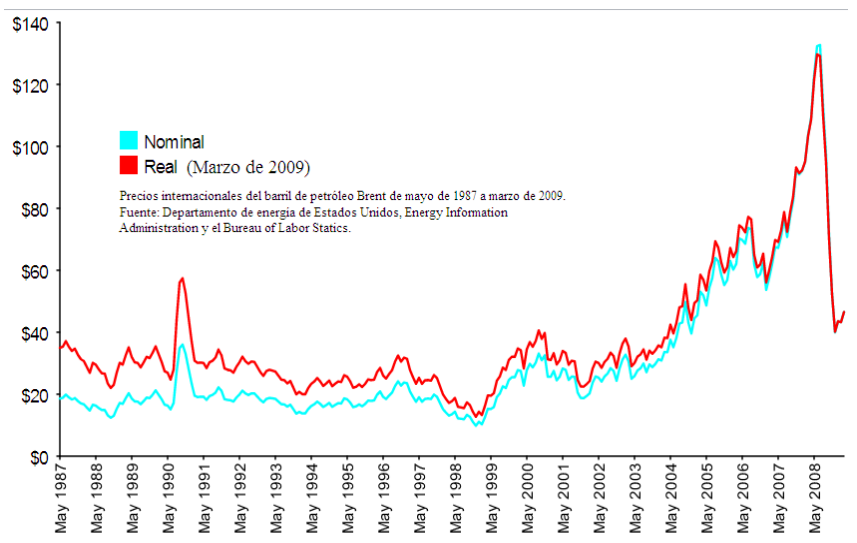

Figura 2: Precio del petróleo Brent

Fuente: Realizado por TomTheHand con datos del organismo público Energy Information Administration

Fue así que se retomaron las aportaciones realizadas en 1883, por el estadounidense Charles Fritts cuando haciendo uso del efecto fotovoltaico estudiado por los físicos Albert Einstein en 1904 y Alexandre-Edmon Becquerel en el año de 1939, fabricó la primera celda fotovoltaica con apenas el $1 \%$ de eficiencia. (Carta-González et al, 2009.) La baja eficiencia en la celda fotovoltaica y la creación de organismos como la OPEP (Organización de Países Exportadores de Petróleo) que regularon la extracción y venta de crudo a nivel mundial, hicieron que la prioridad en la investigación de fuentes de energía renovables fuera menor, así que el presupuesto de investigación para estas nuevas tecnologías se vio limitado. Sin embargo, las fuentes de energía renovables son de suma importancia ya que desde la década de los 80's se lucha contra el calentamiento global y la contaminación que produce la quema de combustibles fósiles, es por eso que ahora se estudia la optimización de estos sistemas para poder dejar de depender de las formas clásicas de generación de energía y realizar una transición energética hacía fuentes más amigables con el medio ambiente. (ECLAC, 1988.) (Carta-González et al, 2009.) (Granda-Gutiérrez et al, 2013.)

\subsection{Sistemas híbridos solares}

En México tenemos un alto potencial para el uso de energías renovables a través de sistemas fotovoltaicos y solares térmicos, ya que nos encontramos en una ubicación geográfica muy conveniente para ello, en la Figura 3 se muestra un mapa de la república mexicana donde se detallan las zonas geográficas en las que el potencial solar fotovoltaico es alto en $\mathrm{kWh} / \mathrm{kWp}$, (kilowatts-hora sobre kilowatts-pico). (SENER, 2012.)

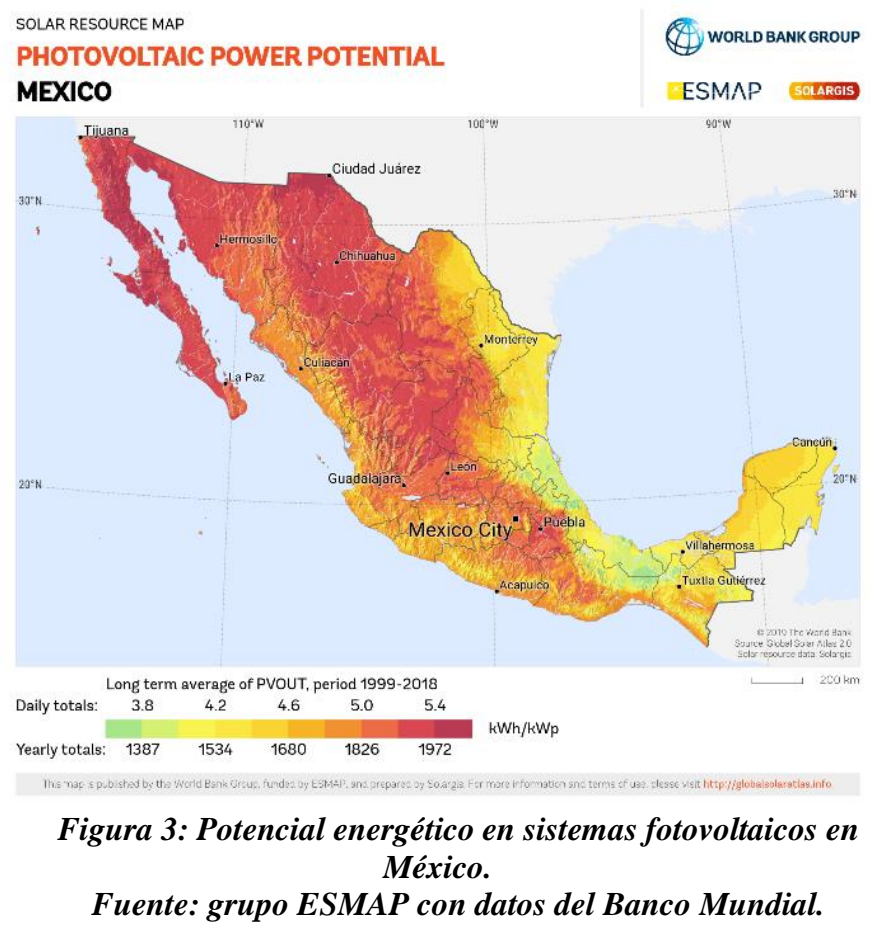

Con el fin de aprovechar una fuente de energía más limpia y amigable con el medio ambiente pero, considerando las limitantes de los sistemas fotovoltaicos como su incapacidad de transformar energía durante la noche o la gran superficie necesaria para su instalación, se ha planteado el uso de otras fuentes y/o tecnologías que trabajen en conjunto, a estos sistemas se les conoce como sistemas híbridos solares, estos sistemas buscan incrementar el desempeño de los sistemas solares fotovoltaicos tradicionales, mediante una transformación en conjunto o auxiliar de la radiación solar u otra fuente energética disponible, sin la desventaja que representa el incremento sustancial de superficie de instalación. En otras palabras, un sistema híbrido solar busca aprovechar de mejor manera los recursos energéticos disponibles. Un ejemplo de estos sistemas es el formado por la interconexión de una celda o panel y generadores termoeléctricos, conocido como sistema hibrido $\mathrm{PV} / \mathrm{TE}$.

\section{Efectos de la temperatura en los sistemas fotovoltaicos $y$ su rendimiento.}

Al exponer una celda fotovoltaica a la radiación solar esta convierte parte de la energía incidente en electricidad aprovechable, sin embargo, otra fracción de esta energía es convertida en calor. De acuerdo a la segunda ley de la 
termodinámica representada en la ecuación 1 , sabemos que la eficiencia de un sistema no puede alcanzar el $100 \%$, traducido en que $\mathrm{Q}_{\mathrm{f}}$ (energía obtenida) tienda a cero, llevando así que el coeficiente de energía obtenida sobre la energía suministrada tienda a cero, obteniendo así que la eficiencia (e) sea 1.

$$
e=1-Q_{f} / Q_{c}
$$

La termodinámica también dicta que todo objeto que aumenta su temperatura sufre de un "reacomodamiento" en sus átomos. En la Figura 4 podemos apreciar este comportamiento, a la izquierda podemos ver el paso de los electrones a través de la estructura a temperatura ambiente, mientras que a la derecha podemos observar que los átomos sufrieron un reacomodamiento, también se muestra que el recorrido de un electrón a través de un material es menor cuando el material se encuentra a temperatura ambiente que cuando este se encuentra sometido a una mayor temperatura.
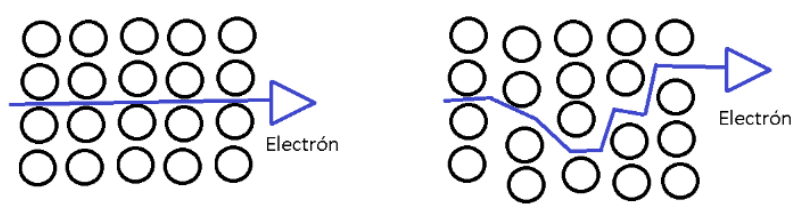
Figura 4: Cambio en la estructura de los átomos por efecto de
la temperatura.

Fuente: Elaboración propia.

Por lo tanto, la corriente eléctrica producida en un sistema fotovoltaico está ligada a la radiación incidente sobre la celda y su temperatura de operación. (Granda-Gutiérrez et al, 2013.), estos son los parámetros que más afectan su rendimiento, y serán los considerados en este estudio.

\section{Metodología experimental}

A continuación se describe la metodología empleada para el análisis del desempeño de un panel fotovoltaico variando sus condiciones de operación. Se propone un sistema hibrido solar que está formado por tres elementos principales, un panel fotovoltaico (PV) de silicio monocristalino de $60 \mathrm{~W}$ cuyas características se muestran en Tabla 1, generadores termoeléctricos TGM-127-1.24-2.5 especificados en la Tabla 2 y Tabla 3, dependiendo de la diferencia de temperatura entre sus placas y un reflector difuso de refuerzo.

Tabla 1: Características del panel fotovoltaico

\begin{tabular}{|l|l|}
\hline Celdas & Silicio monocristalino \\
\hline Potencia de operación & $60 \mathrm{~W}$ \\
\hline $\mathbf{V}_{\text {oc }}$ & $21.5 \mathrm{~V}$ \\
\hline $\mathbf{V}_{\text {op }}$ & $17.2 \mathrm{~V}$ \\
\hline $\mathbf{I}_{\text {op }}$ & 3.48 \\
\hline $\mathbf{I}_{\mathbf{s c}}$ & $4.06 \mathrm{~A}$ \\
\hline Coeficiente de temperatura & $-0.45 \% /{ }^{\circ} \mathrm{C}$ \\
\hline Número de celdas & 24 \\
\hline
\end{tabular}

Fuente: Elaboración propia con datos del fabricante Solartec.
Tabla: 2 Características del generador termoeléctrico con una diferencia de temperatura de $25^{\circ} \mathrm{C}$ entre sus placas.

\begin{tabular}{|c|c|}
\hline $\begin{array}{c}\text { Material de construcción de } \\
\text { los módulos termoeléctricos }\end{array}$ & Telururo de bismuto \\
\hline $\mathbf{V}$ & $0.416 \mathrm{~V}$ \\
\hline $\mathbf{I}$ & $0.19 \mathrm{~A}$ \\
\hline $\mathbf{R}$ & $2.2 \Omega$ \\
\hline
\end{tabular}

Fuente: Elaboración propia con datos de Kryotherm para el modelo TGM-127-1,4-2,5.

Tabla: 3 Características del generador termoeléctrico con una diferencia de temperatura de $35^{\circ} \mathrm{C}$ entre sus placas.

\begin{tabular}{|c|c|}
\hline $\begin{array}{c}\text { Material de construcción de } \\
\text { los módulos termoeléctricos }\end{array}$ & Telururo de bismuto \\
\hline $\mathbf{V}$ & $0.67 \mathrm{~V}$ \\
\hline $\mathbf{I}$ & $0.31 \mathrm{~A}$ \\
\hline $\mathbf{R}$ & $2.26 \Omega$ \\
\hline
\end{tabular}

Fuente: Elaboración propia con datos de Kryotherm para el modelo TGM-127-1,4-2,5.

Inicialmente se analizara el comportamiento del panel de silicio en condiciones ASTMG-173-03, ya que son las condiciones en las que son probados para su venta y distribución. Para ello se parte del comportamiento de una celda fotovoltaica de silicio monocristalino, la cual puede ser representada como un circuito eléctrico, el cual se muestra en la Figura 5.(GrandaGutiérrez et al, 2013.)

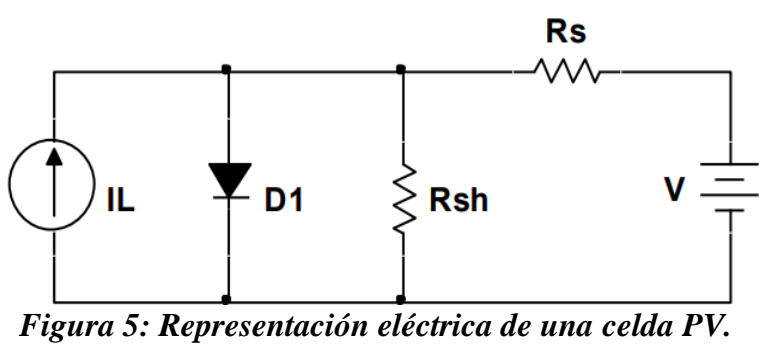

Fuente: (Granda-Gutiérrez et al, 2013)

El diagrama de este circuito puede ser traducido en la siguiente ecuación:

$$
I=I_{L}-I_{0}\left[\exp \left\{\frac{q\left(V+I R_{S}\right)}{K a T_{C}}\right\}-1\right]-I_{S H}
$$

Donde $I$ es la corriente máxima generada por la celda, $I_{L}$ es la corriente fotogenerada, $I_{0}$ la corriente de saturación inversa, $I_{S H}$ la corriente que circula a través de las resistencia en paralelo correspondiente a las uniones existentes entre los elementos, Rs es la resistencia en serie, $R s h$ la resistencia en paralelo; también debemos de considerar las constantes $q$ que representa la carga del electrón, $k$ la constante de Boltzmann, $T c$ la temperatura de operación de la celda y $A$ el factor de idealidad. (Carta-González et al, 2019.) (Granda-Gutiérrez et al, 2013.) (McEvoy, 2012.)

Un panel fotovoltaico es un arreglo en serie y paralelo de celdas fotovoltaicas con la intención de generar un voltaje y corriente adecuados para su uso comercial, tomando como referencia el modelo visto en la ecuación 2, se pueden agregar 
dos nuevos términos que representan el número de celdas en serie (Ns) y paralelo $(\mathrm{Np})$, reescribiéndose de la siguiente manera: (Granda-Gutiérrez et al, 2013.)

$$
I=N p I_{L}-N p I_{0}\left[\exp \left\{\frac{q\left(\frac{V}{N S}+\frac{I R S}{N P}\right)}{K A T_{C}}\right\}-1\right]-I_{S H}
$$

De esta manera, pueden ser agregadas celdas en serie y paralelo según sea necesario a la simulación. Para simular el comportamiento del panel se hace uso del software de Simulink de Matlab. Como primer paso se realiza una representación del circuito eléctrico mostrado en la Figura 5, mediante un diagrama de bloques (ver Figura 6), donde se medirán las variables de interés, las cuales son: irradiancia, temperatura de operación, diferencia de potencial, intensidad de corriente eléctrica y potencia generada así mismo, se introducen los valores de las constantes a utilizar: (O’Mara, 1990.) (Sánchez, 2019.)

Coeficiente de temperatura en condiciones estándar $\mathrm{Ki}=0.0013, \mathrm{q}=1.6 \mathrm{e}-19, \mathrm{~K}=1.38 \mathrm{e}-23, \mathrm{n}=1.3$, Energía de gap dependiente de la temperatura para el silicio $\mathrm{Eg} 0=1.12$, $\mathrm{Rs}=0.221, \mathrm{Rsh}=415.405, \mathrm{Tn}=298, \mathrm{Voc}=21.5, \mathrm{Isc}=4.07, \mathrm{~A}=1.2$

Una vez que los valores de las variables necesarias y los datos de la Tabla 1 son colocados, se procede a realizar la simulación y obtener los valores de las variables de interés.

\section{Corriente fotogenerada $I_{F}$ :}

Para obtener el valor de la corriente de salida del panel es necesario que la simulación resuelva en primera instancia al elemento $\mathrm{NpI}_{L}$ pues este representa el flujo de portadores de carga desplazados por el efecto fotoeléctrico. La corriente fotogenerada por un panel puede obtenerse mediante la ecuación 4 donde para esta primera simulación $\mathrm{G}=1000 \mathrm{~W} / \mathrm{m}^{2}$ y la temperatura de operación de la celda es de $25^{\circ} \mathrm{C}$. Cabe recalcar, que en la práctica, la temperatura de operación bajo estas condiciones es controlada, por lo tanto se mantiene constante.

$$
N p I_{L}=I_{F}=\left[I_{s c}+K_{i} *(T-298)\right] * \frac{G}{1000}
$$

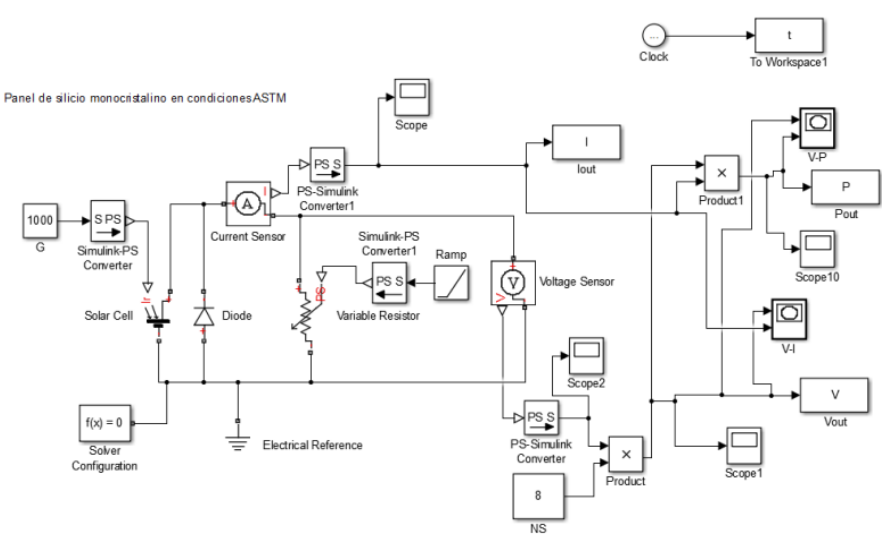

Figura 6: Diagrama de bloques correspondiente a $I_{F}$. Fuente: Elaboración propia.

\section{Corriente de saturación inversa $I_{0}$ :}

Posterior a resolver la corriente fotogenerada, es necesario resolver al elemento $\mathrm{I}_{0}$. La corriente de saturación de un panel puede calcularse mediante la ecuación 5:

$$
N p I_{0}=I_{R S} *\left(\frac{T}{T_{n}}\right)^{3} * \exp \left\{\frac{q * E_{g 0} *\left(\frac{1}{T_{n}}-\frac{1}{T}\right)}{A K T}\right\}
$$

Esta corriente corresponde a la saturación de diodo característica de cada tipo de celda fotovoltaica, para la solución de esta corriente, primero deberá resolverse la variable $I_{R S}$, correspondiente a la resistencia en serie de los elementos del circuito.

\section{Corriente de saturación debido a la resistencia en serie $I_{R S}$ :}

$$
I_{R S}=\frac{I_{S C}}{\exp \left\{N_{S} K T\right\}-1}
$$

Esta corriente suele ser muy pequeña y puede calcularse mediante la ecuación 6 , siendo dependiente de tres características de la celda, la corriente eléctrica que circula por ella, del material semiconductor de fabricación y su temperatura de operación.

Corriente a través de las resistencias debido a las uniones $I_{S H}$ :

$$
I_{S H}=\left(\frac{V+I * R_{S}}{R_{S h}}\right)
$$

Esta corriente puede ser calculada con la ecuación 7, esta es debida a las uniones de soldadura entre los hilos conductores que interconectan las celdas fotovoltaicas y debe de ser restada pues se considera una pérdida del sistema. Al final esta será la responsable de la caída de la corriente en la gráfica.

\section{Desempeño del panel en condiciones ASTMG-173-03:}

Una vez calculadas cada una de las variables anteriormente vistas la ecuación 4 puede ser resuelta. Los resultados de esta primera simulación en condiciones ASTMG-173-03 se muestran a continuación en dos gráficas, la primera muestra el comportamiento en función del voltaje y la corriente de salida (Figura 7), en la segunda, se compara el voltaje de salida contra la potencia generada del panel, permitiendo observar el voltaje al que se alcanza la mayor potencia útil (Figura 8).

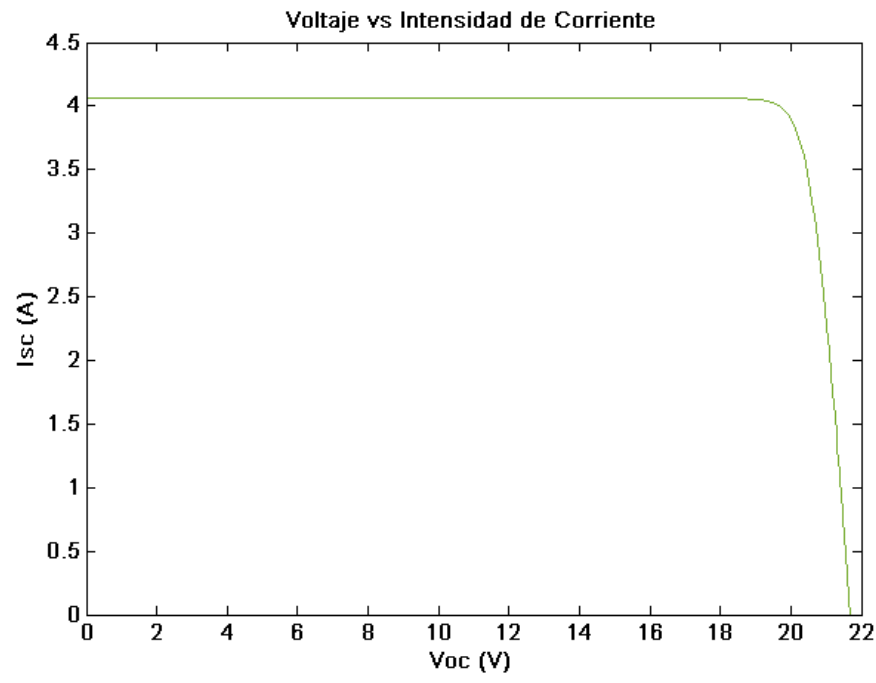

Figura 7: Curva voltaje contra corriente del panel en condiciones ASTMG-173-03 Fuente: Elaboración propia. 


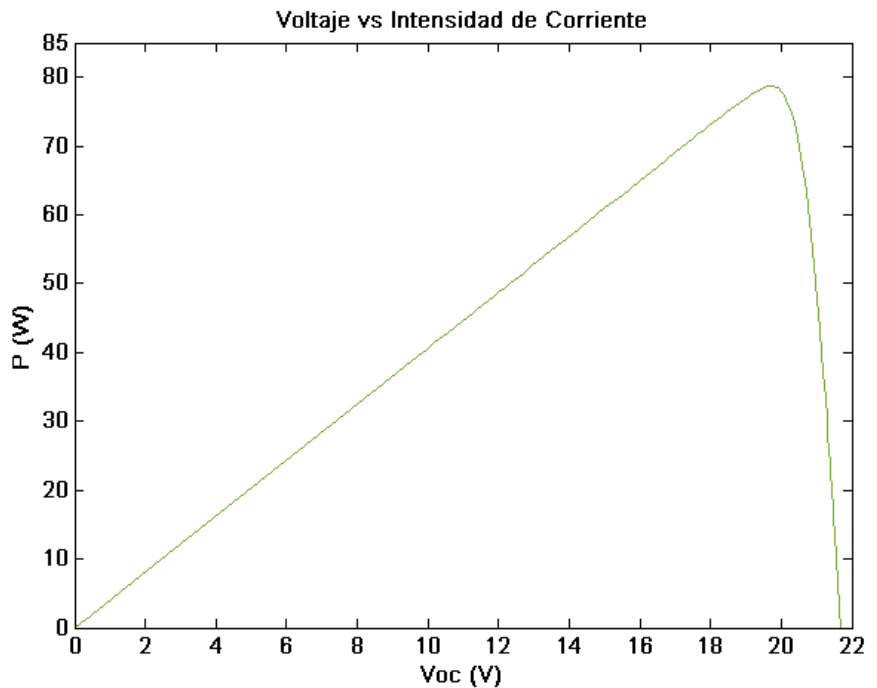

Figura 8: Curva voltaje contra potencia del panel en condiciones ASTMG-173-03

Fuente: Elaboración propia.

En estas gráficas se puede observar que el valor de la diferencia de potencial en las terminales del panel, es de 21.68 $\mathrm{V}$, mientras que el valor de la intensidad de corriente es de 4.07 A y la potencia máxima alcanzada es de $78.7 \mathrm{~W}$.

Desempeño del panel en condiciones $1000 \mathrm{~W} / \mathrm{m}^{2}$ sin refrigeración:

Como se ha mencionado, la temperatura de operación del panel es un factor muy importante a considerar, debido a esto, se plantea una segunda simulación con las mismas condiciones de irradiancia, sin embargo, para esta segunda corrida, la temperatura no se mantiene constante, siendo calculada mediante la ecuación 8 , lo que sería una aproximación más cercana a la realidad de operación. (Nasrin, 2017)

$$
T=2.4308(G)^{0.4352}
$$

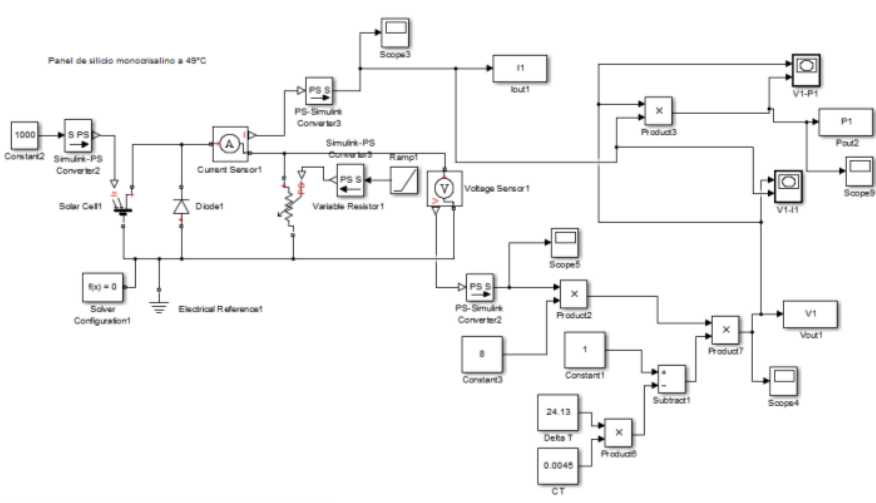

Figura 9: Diagrama de bloques correspondiente a condiciones $1000 \mathrm{~W} / \mathrm{m}^{2}$ sin refrigeración.

Fuente: Elaboración propia.

Con el fin de apreciar los efectos de la temperatura en la conversión eléctrica del panel, y como la radiación incidente juega un papel crucial en ello, se calcula la temperatura de operación a $1000 \mathrm{~W} / \mathrm{m}^{2}$, dando como resultado $49.13{ }^{\circ} \mathrm{C}$, una vez obtenida esta temperatura de operación se ajustan los parámetros de simulación, los cuales se muestran en la Figura 9, los resultados se muestran a continuación.

En la Figura 10 se puede observar una clara disminución en la salida de voltaje del panel debido a la temperatura de operación, esto significa que no únicamente es el voltaje el afectado por el incremento de temperatura, sino que, por ende, la potencia de salida del panel también es afectada, como se observa en la Figura 11.

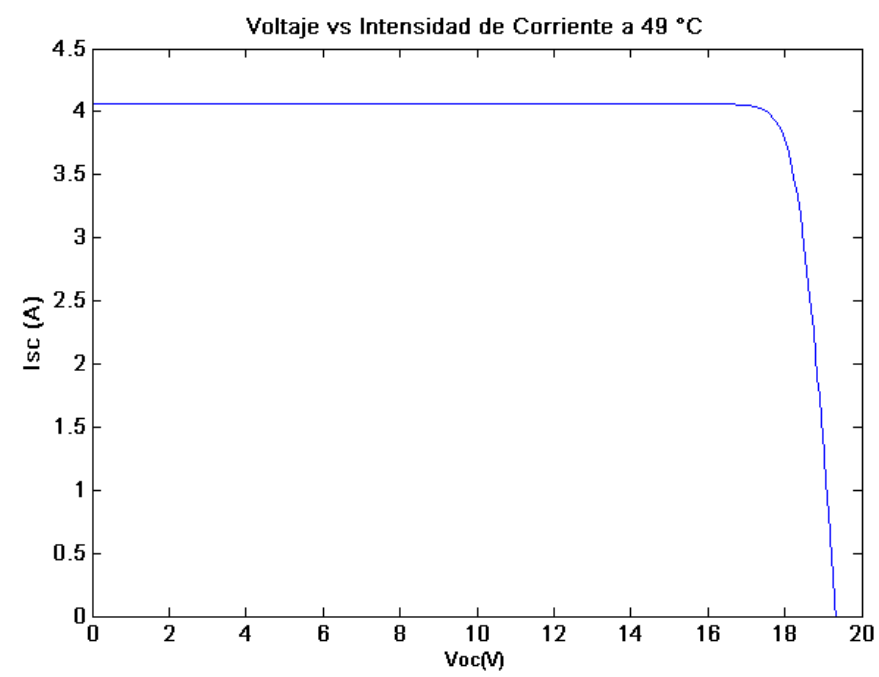

Figura 10: Curva voltaje contra corriente a $49^{\circ} \mathrm{C}$. Fuente: Elaboración propia.

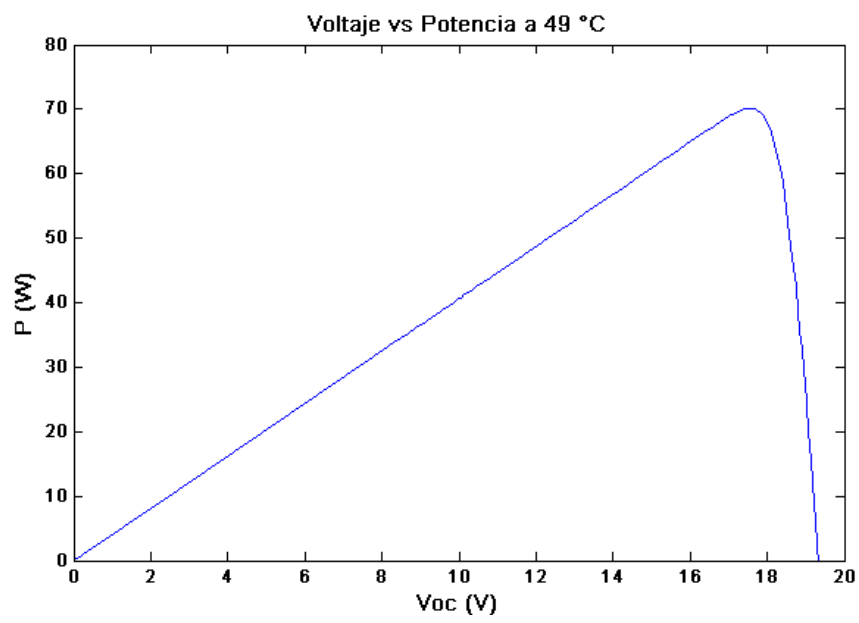

Figura 11: Curva voltaje contra potencia a $49^{\circ} \mathrm{C}$. Fuente: Elaboración propia.

El efecto debido a la temperatura sobre el voltaje y potencia de salida se puede observar con mucha más claridad en la Figura 12 , donde se comparan las curvas de voltaje contra corriente para ambas temperaturas $25^{\circ} \mathrm{C}$ correspondiente a las condiciones de prueba ASTMG-173-03 y $49^{\circ} \mathrm{C}$ en donde la temperatura de operación no es controlada. En esta gráfica se puede observar que el valor en el voltaje de salida cae de 21.68 a $19.32 \mathrm{~V}$. 


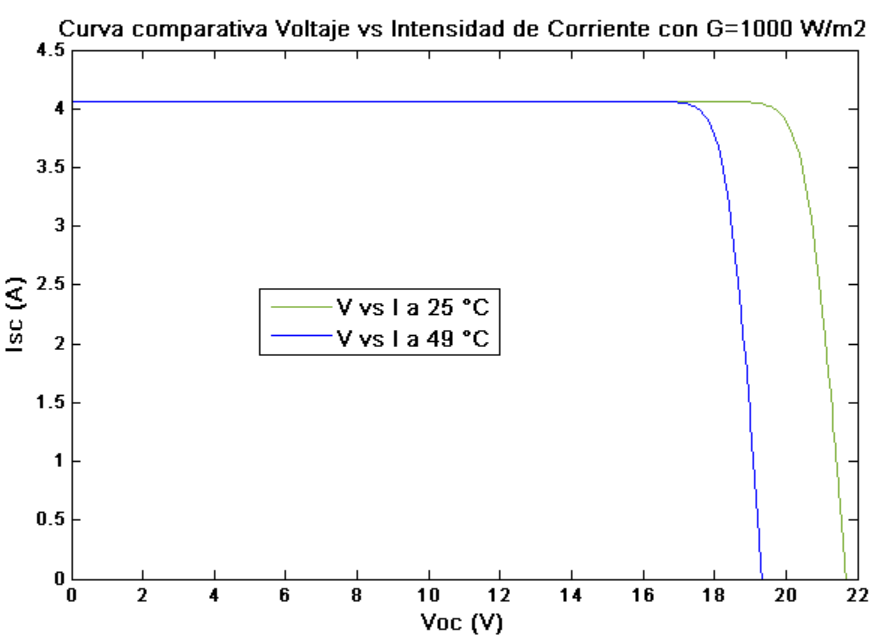

Figura 12: Curva voltaje contra corriente comparativa entre la temperatura de operación a $25{ }^{\circ} \mathrm{C}$ y $49{ }^{\circ} \mathrm{C}$. Fuente: Elaboración propia.

Así mismo, en la Figura 13 se observa la disminución en la generación de potencia debido al incremento de la temperatura de operación.

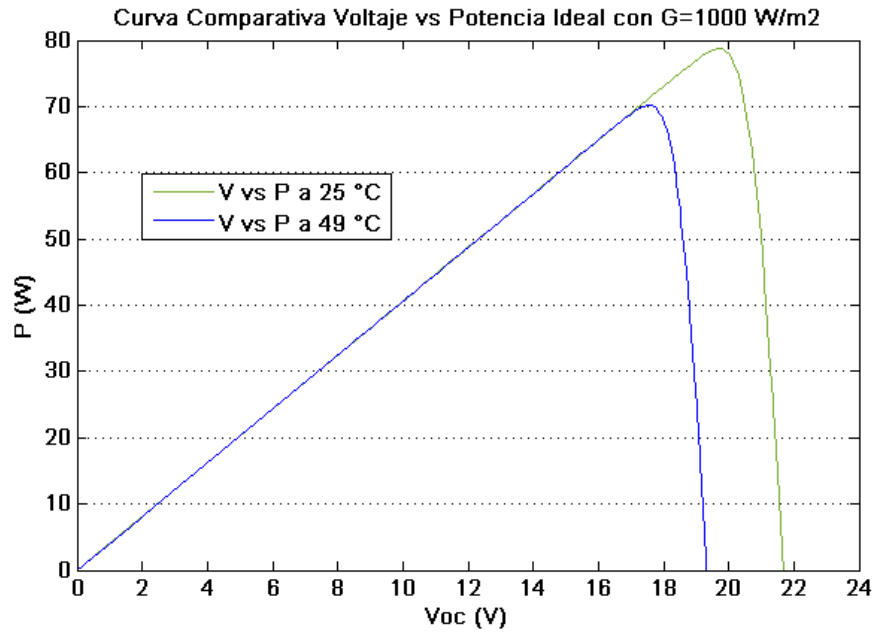

Figura 13: Curva voltaje contra corriente comparativa entre la temperatura de operación a $25^{\circ} \mathrm{C}$ y $49{ }^{\circ} \mathrm{C}$. Fuente: Elaboración propia.

Comparando las curvas de la Figura 13, la potencia baja de 78.7 a $70.15 \mathrm{~W}$ debido al incremento en la temperatura de operación de las celdas que forman el panel.

\section{Desempeño del arreglo Panel + Reflector Difuso:}

Con el fin de estudiar el comportamiento del panel fotovoltaico en presencia de un incremento de irradiancia y la consideración de los efectos del incremento de temperatura inherente a ello, se acopla un reflector difuso de refuerzo experimental a base de un recubrimiento de base cerámica, capaz de incrementar la radiación incidente sobre el panel teóricamente en un $60 \%$, este factor de reflectancia es intrínseco de cada material que funja como recubrimiento en el reflector difuso, por lo tanto, se añade un bloque más a la simulación (Ver Figura 14), que representa un factor equivalente sobre la radiación G, y los resultados se comparan con los obtenidos de las simulaciones del panel fotovoltaico en condiciones ASTMG-173-03 y $1000 \mathrm{~W} / \mathrm{m}^{2}$ sin enfriamiento. Utilizando la ecuación 8 se logra obtener la temperatura de operación la cual se aproxima a $60.28{ }^{\circ} \mathrm{C}$, este parámetro es añadido a la simulación obteniendo lo siguiente:

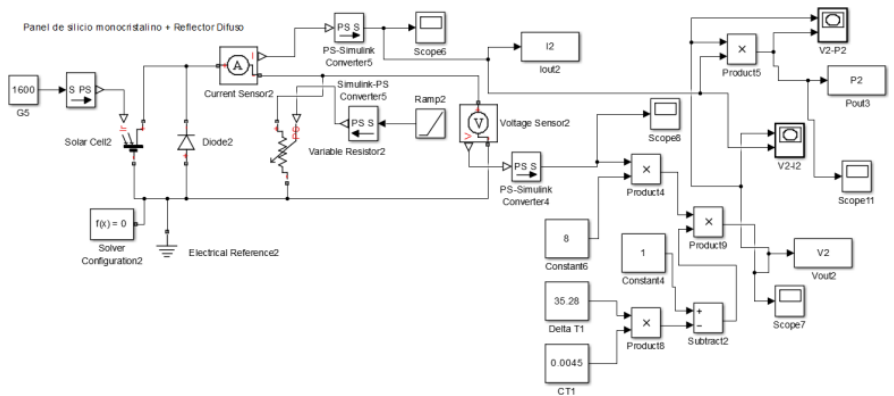

Figura 14: Diagrama de bloques correspondiente al arreglo Panel + Reflector Difuso.

Fuente: Elaboración propia.

En la Figura 15 se puede apreciar que, al incrementarse la radiación incidente sobre el panel, la temperatura se incrementa aproximadamente $11{ }^{\circ} \mathrm{C}$ presentándose una disminución en la generación de 19.23 a $18.36 \mathrm{~V}$ en la diferencia de potencial del panel. Sin embargo, el cambio realmente significativo se puede apreciar al observar la corriente generada en comparación con la simulación anterior, lo cual se ve reflejada en la potencia de salida entregada por el sistema (Figura 16).

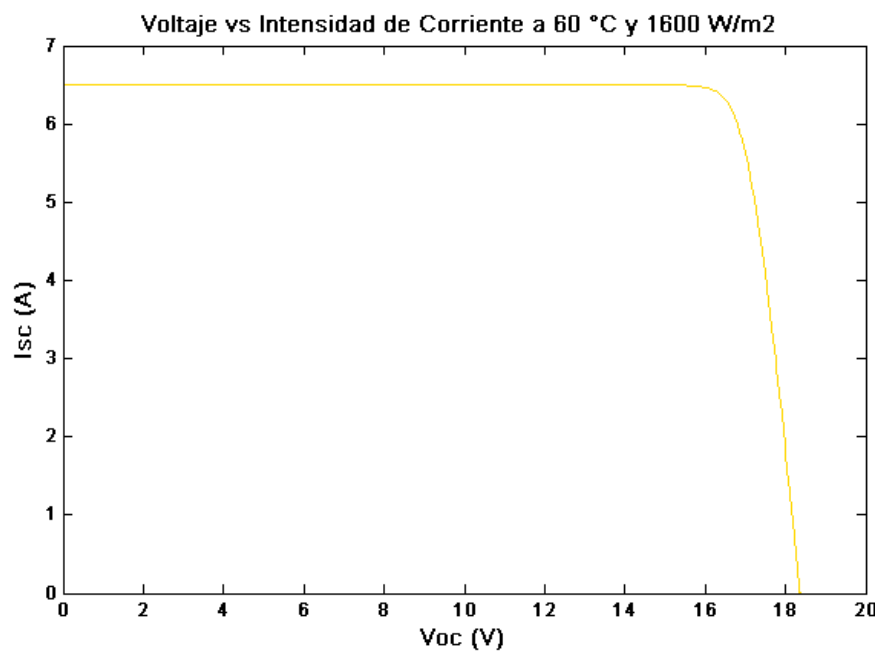

Figura 15: Curva voltaje contra corriente a $60^{\circ} \mathrm{C}$.

Fuente: Elaboración propia.

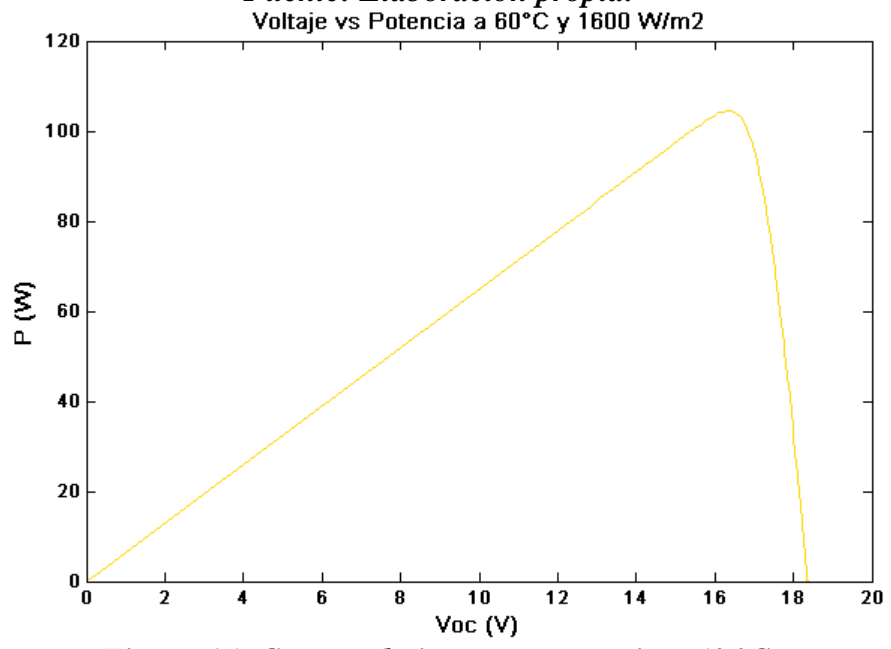

Figura 16: Curva voltaje contra potencia a $60^{\circ} \mathrm{C}$. Fuente: Elaboración propia. 
Este incremento se debe a la mayor incidencia de irradiancia sobre el panel, siendo en esta simulación de $1600 \mathrm{~W} / \mathrm{m}^{2}$ debido a la participación del reflector difuso, lo que según la ecuación 4 , repercute en un incremento en la corriente fotogenerada. El incremento en la corriente de salida va de 4.07 a $6.5 \mathrm{~A}$, lo cual es concurrente con el incremento de irradiancia incidente. Así mismo, el cambio en la potencia generada va de $70.15 \mathrm{~W}$ con una irradiancia de $1000 \mathrm{~W} / \mathrm{m}^{2}$ a $104.4 \mathrm{~W}$ con una irradiancia de $1600 \mathrm{~W} / \mathrm{m}^{2}$ como se observa en la Figura 17.

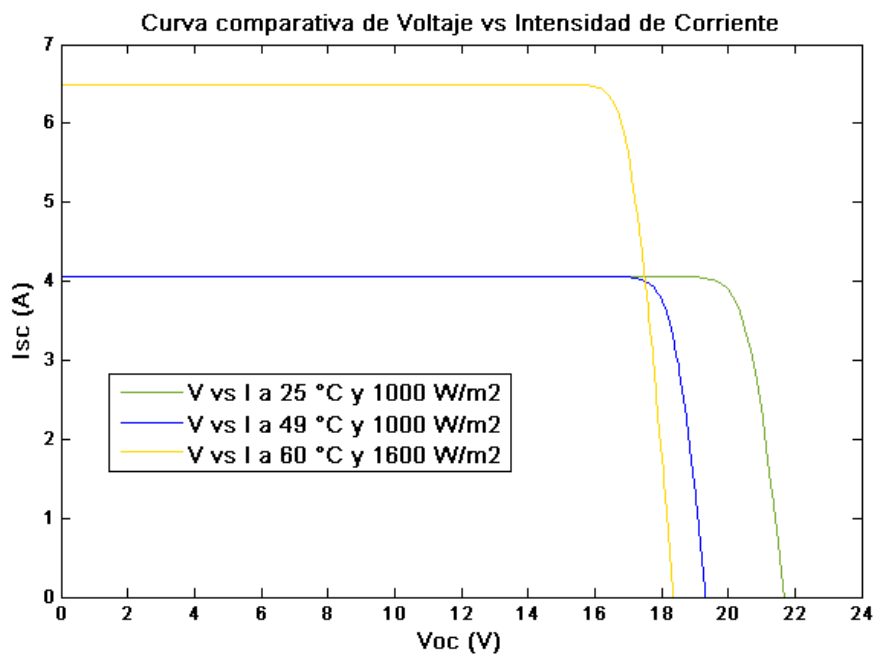

Figura 17: Curva voltaje contra corriente comparativa entre la temperatura de operación a 25,49 y $60{ }^{\circ} \mathrm{C}$.

Fuente: Elaboración propia.

Es importante notar que al incrementar la irradiancia si bien el incremento en la intensidad de corriente es notorio, también lo hace la temperatura de operación del panel, por lo que, la diferencia de potencial a la que se alcanza la máxima potencia útil de salida también es menor, en comparación con las condiciones de operación antes vistas, como se muestra en la Figura 18.

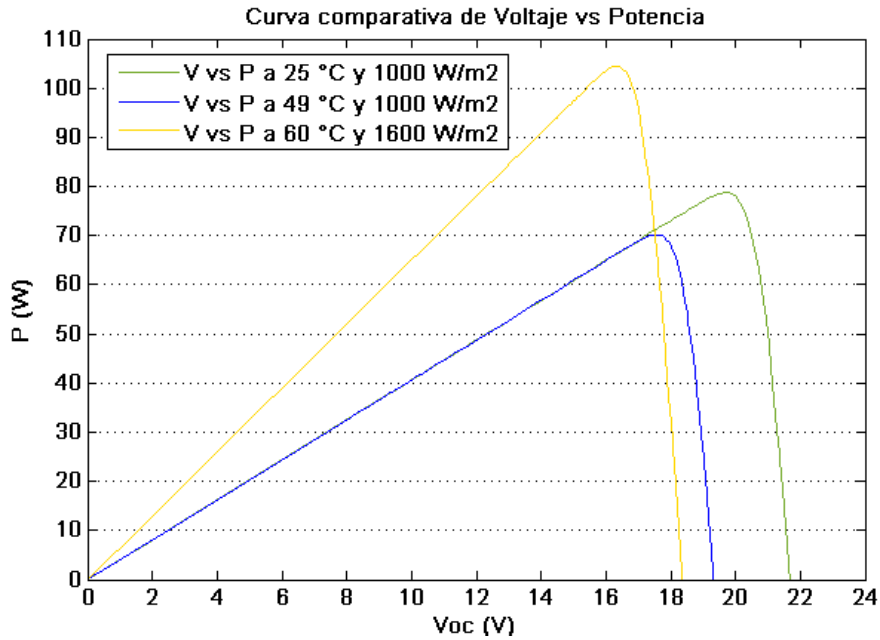

Figura 18: Curva voltaje contra corriente comparativa entre la temperatura de operación 25,49 y $60{ }^{\circ} \mathrm{C}$. Fuente: Elaboración propia.

\section{Desempeño del arreglo Panel + TEG:}

Haciendo la consideración de que un generador termoeléctrico aprovecha la diferencia de temperatura entre dos secciones o placas para generar una diferencia de potencial, y basado en la bibliográfica (Chávez-Urbiola, 2011.) se realiza una simulación donde la corriente y voltaje aportados por los TEGs en un arreglo en serie y paralelo, se ve reflejado en la potencia de salida, a su vez, los resultados también son comparados con los obtenidos del comportamiento del panel en las condiciones de operación ya analizadas.

Utilizando los datos de la Tabla 2; se busca un arreglo de forma que cada celda del panel cuente con una conexión CeldaTEG. Haciendo la consideración de que el panel empleado para la experimentación recibe $1000 \mathrm{~W} / \mathrm{m}^{2}$, su temperatura de operación según la ecuación 8 es de $49.13^{\circ} \mathrm{C}$ y consta de 24 celdas en un arreglo en serie y paralelo, se plantea un arreglo de tres líneas en paralelo cada una con ocho módulos en serie, formando así en su conjunto, un arreglo Panel + TEG, estos datos son agregados a la simulación, como se muestra en la Figura 19, obteniendo los siguientes resultados:

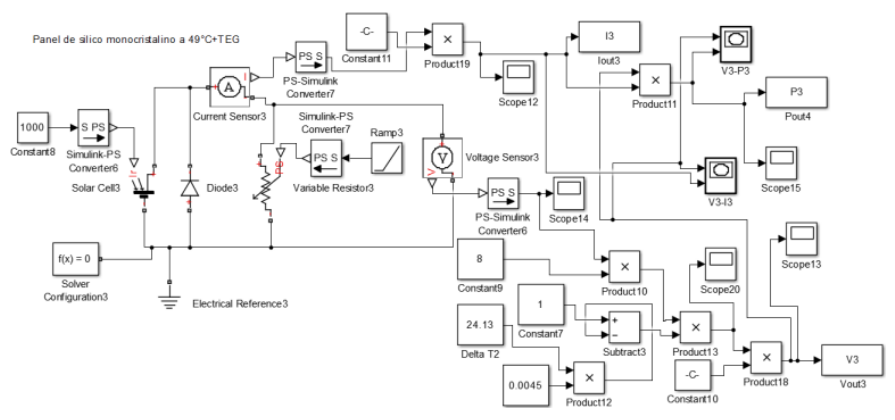

Figura 19: Diagrama de bloques correspondiente al arreglo Panel +TEG

Fuente: Elaboración propia.

En los datos obtenidos de la simulación se puede observar en la Figura 20, la ganancia de voltaje y corriente debido a la acción de los módulos termogeneradores acoplados al panel fotovoltaico, estas ganancias son mucho más apreciables en la Figura 21, donde si se compara el acoplamiento Panel + TEG contra el panel en condiciones de irradiancia igual a $1000 \mathrm{~W} / \mathrm{m}^{2}$ sin enfriamiento, el incremento en el voltaje va de 19.33 a 19.59 V.

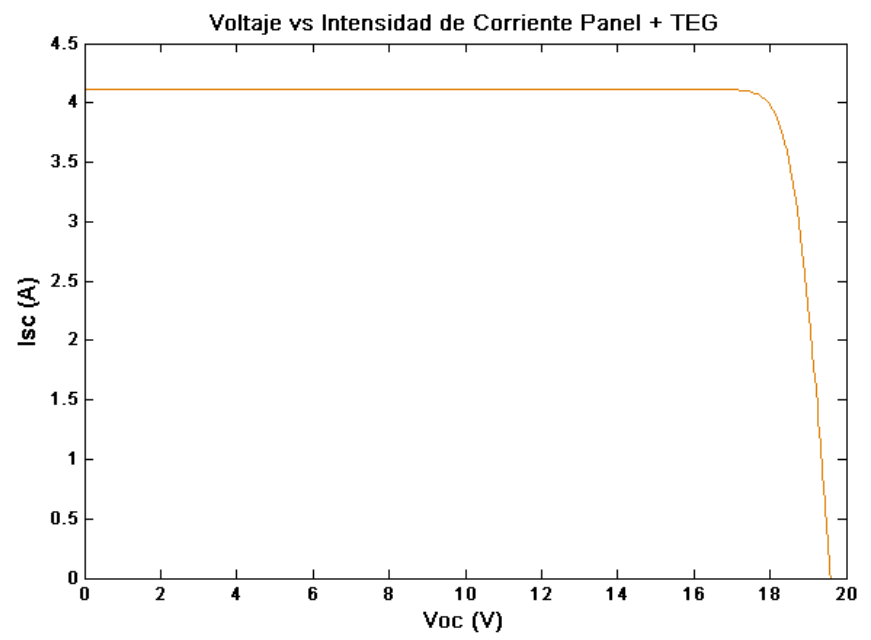

Figura 20: Curva voltaje vs corriente del arreglo panel+ TEG. Fuente: Elaboración propia.

Sin embargo, si bien, existe un incremento en la salida de corriente, si este incremento es comparado con el obtenido mediante el acoplamiento del reflector difuso de refuerzo 
experimental al panel fotovoltaico (ver Figura 21), este incremento es mínimo, ya que va de 4.06 a $4.1 \mathrm{~A}$. El incremento aportado por los generadores termoeléctricos, se ve afectado por la diferencia de temperatura existente entre las secciones fría y caliente de los mismos. Para esta simulación se logra apreciar que el voltaje al cual se alcanza la máxima potencia útil del panel apenas es poco mayor que en el caso $1000 \mathrm{~W} / \mathrm{m}^{2}$ sin enfriamiento por lo que no parece haber una diferencia notoria. Lo mismo sucede si es comparado con respecto al arreglo Panel + Reflector Difuso donde la diferencia entre ambos puntos de aproximadamente de $2 \mathrm{~V}$.

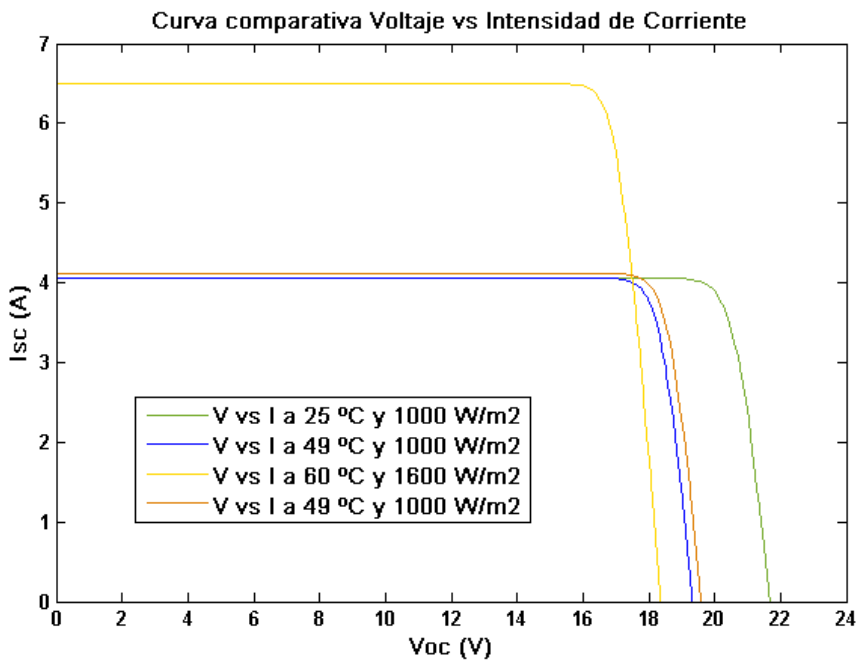

Figura 21: Curva voltaje contra corriente comparativa entre la temperatura de operación 25,49 y $60^{\circ} \mathrm{C}$, las irradiancias de 1000 y $1600 \mathrm{~W} / \mathrm{m}^{2}$ y el acoplamiento Panel + TEG. Fuente: Elaboración propia.

Realizando una comparación de la potencia total de salida del arreglo Panel + TEG mostrada en la Figura 22 donde alcanza una potencia máxima de $72.06 \mathrm{~W}$ contra los $70.15 \mathrm{~W}$ que se presentan en condiciones a $1000 \mathrm{~W} / \mathrm{m}^{2} \sin$ refrigeración.

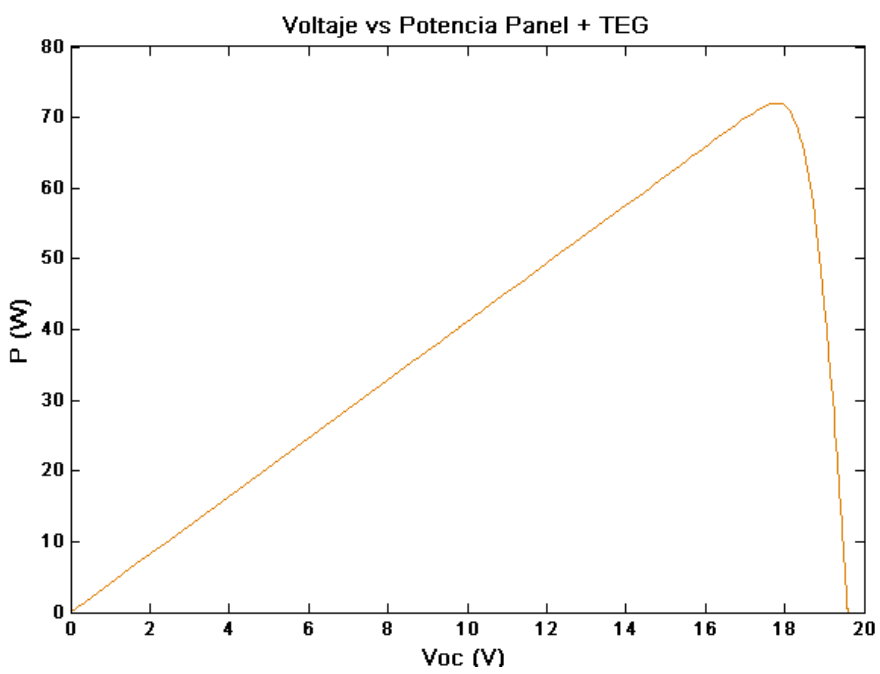

Figura 22: Curva voltaje vs potencia del arreglo panel + TEG. Fuente: Elaboración propia.

El incremento en la potencia al igual que la corriente palidecen si se les compara con los otorgados por el acoplamiento Panel + Reflector Difuso, donde si bien el voltaje proporcionado por este arreglo es menor, la corriente y su efecto sobre la potencia de salida es notoriamente mayor, en la Figura 23, este efecto puede apreciar de forma más clara.

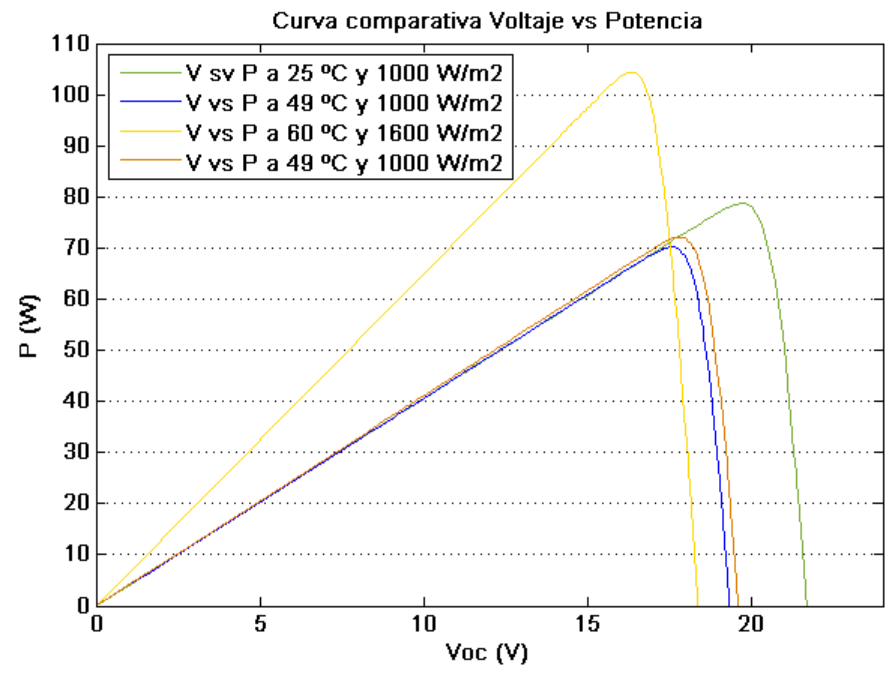

Figura 23: Curva voltaje contra potencia comparativa entre la temperatura de operación 25,49 y $60^{\circ} \mathrm{C}$, las irradiancias 1000 y $1600 \mathrm{~W} / \mathrm{m}^{2}$ y el acoplamiento Panel + TEG. Fuente: Elaboración propia.

Desempeño del arreglo Panel + TEG + Reflector Difuso:

Dado que las simulaciones anteriores muestran un incremento en el rendimiento del panel fotovoltaico, se acoplan en un solo sistema tanto el reflector difuso de refuerzo como el arreglo TEG, esta vez con los datos de la Tabla 3 debido al incremento de irradiancia responsable del incremento de temperatura, con el objetivo de analizar el desempeño de este sistema híbrido como se muestra en la Figura 24.

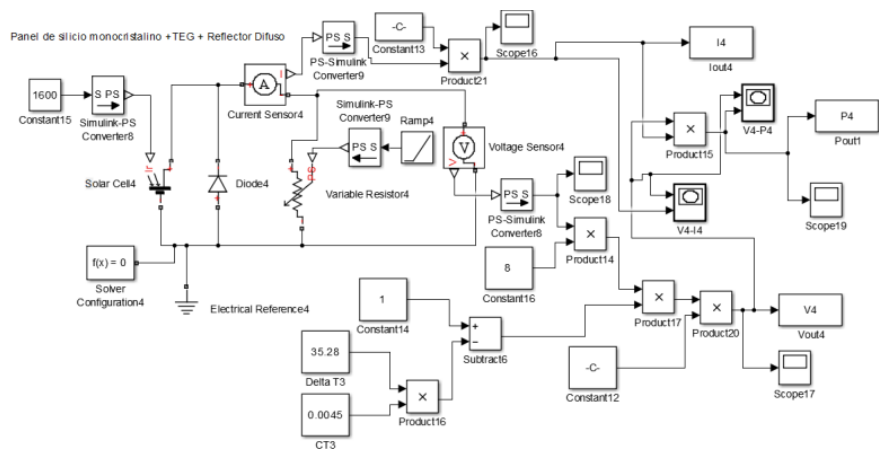

Figura 24: Diagrama de bloques correspondiente al arreglo Panel + TEG + Reflector Difuso

Fuente: Elaboración propia.

En los resultados otorgados por la simulación se puede apreciar un incremento en la transformación tanto de corriente como de voltaje a la salida del sistema (ver Figura 25), este comportamiento era esperado y a pesar del incremento en la temperatura, la caída en la generación de voltaje no es tan sustancial comparándola con la producción obtenida al acoplar únicamente el reflector difuso, esto se debe a la incorporación de los generadores termoeléctricos, los cuales al aprovechar el incremento de temperatura entre sus placas, el cual en este arreglo es mayor que el existente en el arreglo Panel + TEG debido que al incidir mayor cantidad de irradiancia gracias al reflector difuso, existe un incremento en la temperatura de 
operación y por ende un mayor gradiente de temperatura entre las placas que al transformarlo en una diferencia de potencial adicional, el efecto de la temperatura en la producción eléctrica se ve mermado. Este comportamiento estable se puede apreciar en las Figuras 25 y 27.

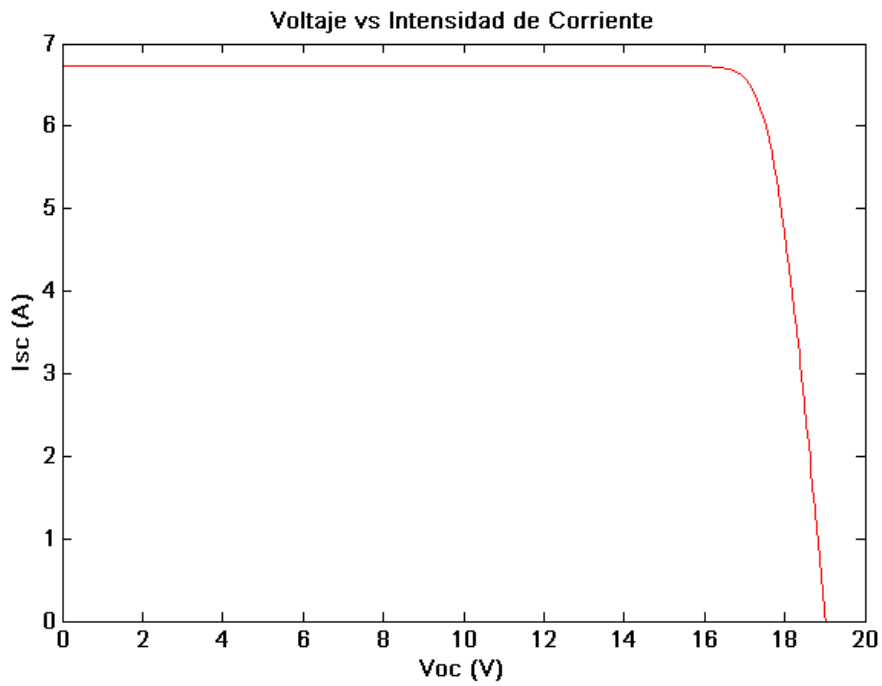

Figura 25: Curva voltaje contra corriente arreglo Panel + TEG + Reflector Difuso.

Fuente: Elaboración propia.

De igual manera que sucede con el voltaje y la corriente eléctrica, el incremento en la potencia es uniforme y se observa un muy claro incremento en la potencia de salida del sistema mostrado en la Figura 26, este sustancial incremento se debe a la mayor cantidad de irradiancia recibida sobre el panel y a la aportación del arreglo Panel + TEG que aprovecha el incremento de temperatura debido a la mayor incidencia de radiación.

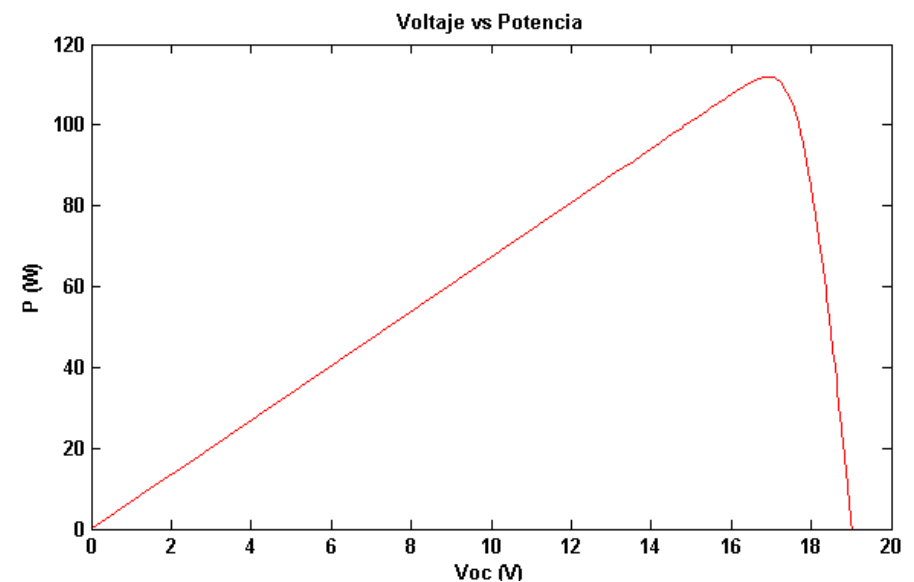

Figura 26: Curva voltaje contra potencia del arreglo Panel + TEG + Reflector Difuso.

Fuente: Elaboración propia.

En la Figura 27 se puede que observar que existe un incremento en el voltaje y corriente de salida, no obstante, el incremento no luce significativo al considerarse un solo panel. Sin embargo, se aprecia que el voltaje al que se alcanza la máxima potencia es prácticamente el mismo que en las condiciones de operación $1000 \mathrm{~W} / \mathrm{m}^{2}$ sin refrigeración, lo que significa que alcanzar una mayor potencia de salida al mismo voltaje.

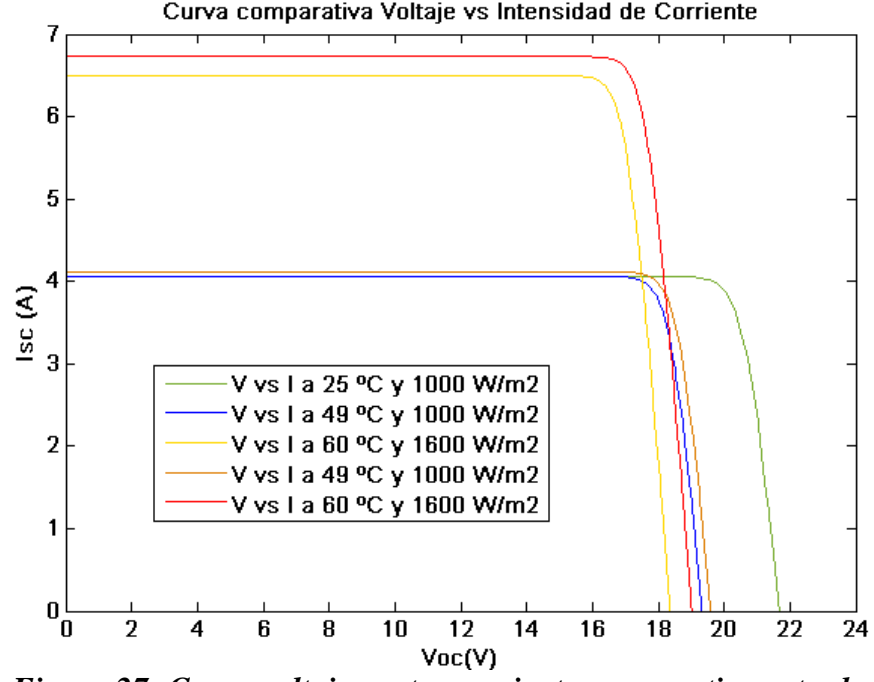

Figura 27: Curva voltaje contra corriente comparativa entre la temperatura de operación 25,49 y $60^{\circ} \mathrm{C}$, las irradiancias 1000 y $1600 \mathrm{~W} / \mathrm{m}^{2}$, el acoplamiento Panel + TEG y el arreglo Panel + TEG + Reflector Difuso.

Fuente: Elaboración propia.

Finalmente, se unen las gráficas generadas a partir de las simulaciones previas y esta nueva propuesta, con el fin de comparar los datos de cada una de ellas en una sola gráfica, se observa en la Figura 28, que el ensamble Panel + TEG + Reflector Difuso es el arreglo con mayor rendimiento de los propuestos.

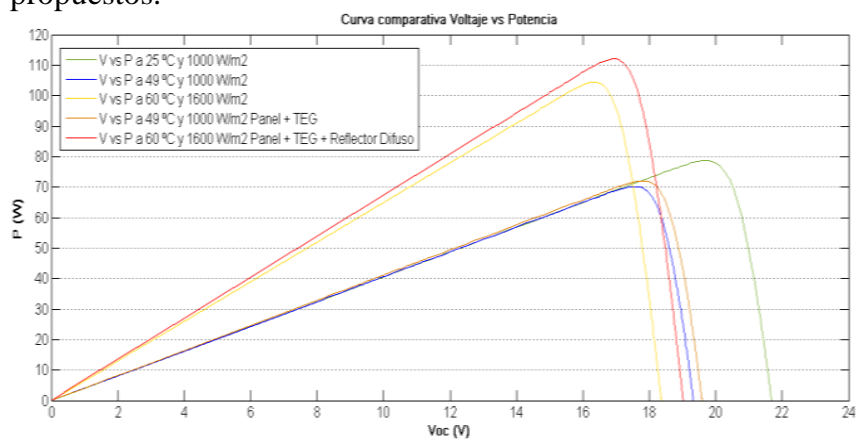

Figura 28: Curva voltaje contra potencia comparativa entre la temperatura de operación 25,49 y $60{ }^{\circ} \mathrm{C}$, las irradiancias 1000 y $1600 \mathrm{~W} / \mathrm{m}^{2}$, el acoplamiento Panel + TEG y el arreglo Panel + TEG + Reflector Difuso.

Fuente: Elaboración propia.

\section{Resultados}

En la Tabla 4 se muestran los resultados de todas las simulaciones realizadas y comparadas para este estudio. En esta se pueden comparar los valores de voltaje, corriente y potencia de salida de casa una de ellas.

Se puede observar que si se compara el desempeño en condiciones ASTMG-173-03 contra $1000 \mathrm{~W} / \mathrm{m}^{2}$ sin refrigeración $\left(49^{\circ} \mathrm{C}\right)$, el valor en el voltaje de salida cae de 21.68 a $19.32 \mathrm{~V}$, lo que representa una caída del $10.88 \%$, de igual manera, la potencia baja de 78.7 a $70.15 \mathrm{~W}$, lo que representa un $10.94 \%$ de potencia total producida debido al incremento en la temperatura de operación de las celdas que forman el panel, sin embargo, esta última condición de es más próxima a su operación real, por lo que es la condición más relevante a considerar. 
Si se compara el PV en condiciones $1000 \mathrm{~W} / \mathrm{m}^{2}$ sin refrigeración $\left(49^{\circ} \mathrm{C}\right)$ contra el arreglo Panel + Reflector Difuso, se puede apreciar una disminución en la generación de 19.23 a $18.36 \mathrm{~V}$, lo que significa una reducción del $4.52 \%$. No obstante existe un incremento en la corriente de salida que va de 4.07 a 6.5 A, lo que representa un incremento del $59.7 \%$, lo cual es concurrente con el incremento de irradiancia incidente. Así mismo, el cambio en la potencia generada va de $70.15 \mathrm{~W}$ con una irradiancia de $1000 \mathrm{~W} / \mathrm{m}^{2}$ a $104.4 \mathrm{~W}$ con una irradiancia de $1600 \mathrm{~W} / \mathrm{m}^{2}$ representando del $48.82 \%$ en la producción total.

Tabla 4: Comparativa del desempeño de un PV bajo diferentes condiciones de operación.

\begin{tabular}{|c|c|c|c|}
\hline Arreglo/condición & $\begin{array}{c}\text { Voltaje a } \\
\text { circuito } \\
\text { abierto } \\
\text { (V) }\end{array}$ & $\begin{array}{c}\text { Corriente } \\
\text { a corto } \\
\text { circuito } \\
\text { (A) }\end{array}$ & $\begin{array}{c}\text { Potencia } \\
\text { (W) }\end{array}$ \\
\hline $\begin{array}{c}\text { ASTMG-173-03 } \\
\left(1000 \mathrm{~W} / \mathrm{m}^{2}-25^{\circ} \mathrm{C}\right)\end{array}$ & 21.68 & 4.06 & 78.7 \\
\hline $\begin{array}{c}1000 \mathrm{~W} / \mathrm{m}^{2} \text { sin } \\
\text { refrigeración } \\
\left(49^{\circ} \mathrm{C}\right)\end{array}$ & 19.32 & 4.05 & 70.15 \\
\hline $\begin{array}{c}\text { Panel }+ \text { TEG } \\
\left(1000 \mathrm{~W} / \mathrm{m}^{2}-49^{\circ} \mathrm{C}\right)\end{array}$ & 19.59 & 4.1 & 72.06 \\
\hline $\begin{array}{c}\text { Panel }+ \text { Reflector } \\
\text { Difuso } \\
\left(1600 \mathrm{~W} / \mathrm{m}^{2}-60^{\circ} \mathrm{C}\right)\end{array}$ & 18.36 & 6.5 & 104.4 \\
\hline $\begin{array}{c}\text { Panel }+\mathrm{TEG}^{\mathrm{Reflector} \text { Difuso }} \\
\left(1600 \mathrm{~W} / \mathrm{m}^{2}-60^{\circ} \mathrm{C}\right)\end{array}$ & 19.02 & 6.73 & 112 \\
\hline
\end{tabular}

Fuente: Elaboración propia.

Si se compara el acoplamiento Panel + TEG contra el panel en condiciones de irradiancia igual a $1000 \mathrm{~W} / \mathrm{m}^{2}$ sin enfriamiento, el incremento en el voltaje va de 19.33 a $19.59 \mathrm{~V}$, representando un incremento del 1.34\%. Sin embargo, si bien, existe un incremento en la salida de corriente este es de solo del 1.13\%, por lo cual, luce minimizado en comparación con el aporte realizado por un reflector difuso. Realizando una comparación de la potencia total de salida, se alcanza una potencia máxima de $72.06 \mathrm{~W}$ contra los $70.15 \mathrm{~W}$ que se presentan en condiciones a $1000 \mathrm{~W} / \mathrm{m}^{2} \sin$ refrigeración, el incremento en la potencia total de salida representa un incremento de apenas el $2.72 \%$.

Finalmente comparando el arreglo Panel + TEG + Reflector Difuso con el generado utilizando un panel en solitario a 1000 $\mathrm{W} / \mathrm{m}^{2}$ sin refrigeración, el incremento en potencia va de 70.15 a $112 \mathrm{~W}$, lo que representa un incremento total de $59.65 \%$ y de $7.28 \%$ si se le compara con el ensamble Panel + Reflector Difuso, con respecto a la intensidad de corriente va de 4.07 a $6.73 \mathrm{~A}$, incrementando así $65.35 \%$, finalmente, dado el incremento de insolación sobre el sistema debido al efecto del relector difuso, el panel incrementa su temperatura, por lo que la conversión en voltaje se ve reducida de 19.23 a 19.02, lo que representa una reducción del $1.09 \%$, sin embargo, si se compara con el acoplamiento Panel + Reflector Difuso, donde el voltaje máximo de salida es de $18.36 \mathrm{~V}$, el acoplamiento extra de TEGs incrementaría el voltaje de salida del sistema en un $3.59 \%$.

\section{Discusión}

Realizando un análisis de los datos de las simulaciones realizadas, se observa que el desempeño de un sistema fotovoltaico puede incrementarse con la interconexión de generadores termoeléctricos y sobre todo, con el acoplamiento de un reflector difuso de refuerzo. Para realizar una comparativa con los resultados presentados por otros autores, es necesario aclarar que estos resultados suelen presentarse en condiciones ASTMG-173-03 cuando a eficiencia de los paneles se refiere. En la Tabla 5 se pueden observar los resultados mencionados.

Tabla 5: Comparativa de los resultados de simulación de este estudio y los de otros autores.

\begin{tabular}{|c|c|c|c|c|}
\hline $\begin{array}{c}\text { Material } \\
\text { del PV }\end{array}$ & $\begin{array}{c}\text { Material } \\
\text { del TEG }\end{array}$ & $\begin{array}{c}\text { Eficiencia } \\
\text { del PV } \\
(\%)\end{array}$ & $\Delta \mathbf{T}\left({ }^{\circ} \mathbf{C}\right)$ & Fuente \\
\hline Silicio-c & - & 16 & 0 & NREL \\
\hline Silicio-c & - & 14.26 & $24^{*}$ & $\begin{array}{c}\text { Este } \\
\text { estudio }\end{array}$ \\
\hline Silicio-c & $\mathrm{Bi}_{2} \mathrm{Te}_{3}$ & 16.3 & 15 & Park \\
\hline Silicio-c & $\mathrm{Bi}_{2} \mathrm{Te}_{3}$ & 16.62 & 24 & $\begin{array}{c}\text { Este } \\
\text { estudio }\end{array}$ \\
\hline $\begin{array}{c}\text { Silicio-c } \\
\mathrm{Bi}_{0.4} \mathrm{Sb}_{1.6} \mathrm{Te}_{3}\end{array}$ & 18.6 & - & Zhang \\
\hline $\begin{array}{c}\text { Silicio-c }+ \\
\text { Reflector } \\
\text { de base } \\
\text { cerámica }\end{array}$ & - & 23.8 & 0 & $\begin{array}{c}\text { Este } \\
\text { estudio }\end{array}$ \\
\hline $\begin{array}{c}\text { Silicio-c }+ \\
\text { Reflector } \\
\text { de base } \\
\text { cerámica }\end{array}$ & - & 21.22 & $35^{*}$ & $\begin{array}{c}\text { Este } \\
\text { estudio }\end{array}$ \\
\hline $\begin{array}{c}\text { Silicio-c }+ \\
\text { TEG + } \\
\text { Reflector } \\
\text { de base } \\
\text { cerámica }\end{array}$ & $\mathrm{Bi}_{2} \mathrm{Te}_{3}$ & 22.77 & $35^{*}$ & $\begin{array}{c}\text { Este } \\
\text { estudio }\end{array}$ \\
\hline \begin{tabular}{c} 
Funcionation \\
\hline
\end{tabular} & & & \\
\hline
\end{tabular}

*Funcionamiento en condiciones de operación simuladas.

Fuente: Elaboración propia con datos de Huen, 2017.

Como se logra apreciar, los resultados obtenidos en las simulaciones son similares a los datos reportados por otros autores, como es el caso del sistema Panel + TEG. Así mismo se logra apreciar que el acoplamiento de un reflector difuso incrementa significativamente la generación de energía.

\section{Conclusiones}

Tomando en cuenta todos los datos obtenidos de cada una de las simulaciones, se concluye que, con el objetivo de incrementar el rendimiento de sistemas fotovoltaicos, el acoplamiento en solitario de generadores termoeléctricos en un sistema hibrido $\mathrm{PV} / \mathrm{TE}$ representa un incremento muy pequeño en la potencia de salida, no obstante, el acoplamiento de reflectores difusos experimentales a base de un recubrimiento de base cerámica, resulta una opción viable para incrementar el desempeño de los sistemas fotovoltaicos, dado su bajo costo en comparación con el de los generadores termoeléctricos y sobre todo, debido al sustancial incremento en la potencia de generación. También se concluye que la aplicación de reflectores difusos de refuerzo puede ser una alternativa a la concentración óptica típicamente empleada en los sistemas híbridos PV/TE, cabe mencionar que el utilizar en simultaneo tanto generadores termoeléctricos y un 
reflector difuso, resulta en una opción válida en la búsqueda de mejorar el desempeño de sistemas fotovoltaicos.

\section{Agradecimientos}

Se agradece al CONACyT por el financiamiento mediante el proyecto 282260 "Incremento del desempeño de sistema solar híbrido utilizando reflectores de refuerzo difusos" perteneciente a la convocatoria de ciencia básica 2016. También agradeciendo a los familiares y colaboradores de cada uno de los autores por su apoyo en cada una de las etapas de esta investigación.

\section{Referencias}

Carta-González, J.A., Calero-Pérez, R., Colmenar-Santos, A., ManuelAlonso-Castro, G., (2009). Centrales de Energías Renovables: Generación Eléctrica con Energías Renovables. Pearson Education, S.A., Madrid, España.

Chávez-Urbiola, E.A., (2011). Solar hybrid systems with thermoelectric generators, Solar Energy-Elsevier, 369-378, Paises Bajos. DOI:10.1016/j.solener.2011.10.020
Economic Commission for Latin America and the Caribbean (ECLAC), (1988). Cambios en la inserción de América Latina en el mercado mundial de energéticos. United Nations 4-72.

Granda-Gutiérrez, E.E., Orta, O.A., Díaz-Guillén, J.C., Jímenez, M.A., Osorio, M., González, M.A., (2013). Modelado y simulación de celdas solares. Congr. Int. Ing. Electrón. Mem. Electro 2013, 17-22. ISSN 1405 2172.

McEvoy, A., Markvart, T., Castañer, L. (2012). Practical Handbook of Photovoltaics Fundamentals and Applicatios. Academic Press-Elsevier, Oxfort, UK.

National Renewable Energy Laboratory (NREL), Best Research-Cell Efficienies, 15013 Denver W Pkwy, Golden, CO 80401, Estados Unidos.

Priscilla Huen, Walid A. Daoud, (2017), Advances in hybrid solar photovoltaic and thermoelectric generators, Renewable and Sustentable Energy Reviews, 1295-1302, Países Bajos.

O'Mara, W.C., Herring, R.B., Hunt, L.P., (1990). Handbook of semiconductor silicon technology. Noyes Publications, New Jersey, USA. ISBN:0-8155-1237-6.

Park, K.-T., Shin, S.-M., Tazebay, A.S., Um, H.-D., J.-Y., Jee, S.-Wlee, J.H., (2013), Lossless hybridization between photovoltaic and thermoelectric devices, Scientific Reports, London, UK.

Sánchez-Fraile, M., (2019). Desarrollo de una herramienta para el modelado del comportamiento eléctrico de células solares. Universidad de Alcalá, Alcalá, España.

Secretaria de Energía (SENER), (2020). Programa Sectorial de Energía 2020-2024. Gobierno de México, México.

Zhang, J., Xuan, Y., \& Yang, L. (2014). Performance estimation of photovoltaic-thermoelectric hybrid systems. Energy, Paises Bajos. 